\title{
Le fortificazioni della Toscana Tirrenica: metodologie e fonti dell'indagine geo-storica
}

\section{Leonardo Rombai}

History, Archaeologic, Geography, Fine and Performing Arts Department, University of Florence, Italy, leonardo.rombai@unifi.it

\begin{abstract}
The research about Tyrrhenian Tuscany fortifications - more than 150 structures studied with Anna Guarducci and Marco Piccardi - is a multidisciplinary and complex topic that requires methods and sources typical of geographical-historical research. This because of various environmental and landscape peculiarity of Tyrrhenian Tuscany and because of her historical sequence from Medieval Age to nowadays. In Tyrrhenian Tuscany - thanks to its strategic and business importance - although low population, cereal and pastoral "latifondo", the marshes and few settlements, a dense fortifications system was gradually developed until the unification of Italy. This study starts from the first researches on Maremma fortifications on Seventies-Eighties of last century, and they also begins from following studies concerning all coastal area or single places, buildings and small areas. This research take into consideration those researchs methods and subjects. But because of the study area extension a larger range of printed and unpublished sources was analyzed. The set of documents was composed by maps, iconographies, photographies, aerial photographies, territorial and literaly studies, etc. The political and administrative fragmentation of Tyrrhenian Tuscany explains the position of the sources (about fortifications) in libraries and archives of Tuscany (Massa, Lucca, Firenze, Pisa, Livorno, Piombino, Siena, Grosseto, Orbetello), of Italy (Genova, Modena, Roma e Napoli) and of foreign countries (Simancas, Parigi, Vienna e Praga); and in municipal and state or authorithy archives too. The partition expalains also the local and regional nature of the most studies. All these documents are often thematic and incomplete, and have been completed with the study of the present contexts (toponyms, buildings and direct evidences of inhabitants), in order to identify and locate the fortifications. This comparative analysis, together with measurements allowed by cartography, has allowed the recognition of the position of more than 30 towers have now disappeared or reduced to ruin.
\end{abstract}

Keywords: Fortifications, Tyrrhenian Tuscany, Historical Geography, methods, sources of investigations

\section{Introduzione}

Il lavoro, insieme con quello di Anna e finalizzato alla conoscenza, fruizione e Guarducci, allarga l'indagine sul patrimonio storico cartografico-iconografico e la storia territoriale della Toscana tirrenica effettuate nel progetto europeo transfrontaliero PERLA (ProgEtto peR l'Accessibilità, la fruibilità e la sicurezza della fascia costiera delle regioni transfrontaliere), conclusosi a fine 202 valorizzazione delle coste toscane e mediterranee. Il gruppo di ricerca - Anna Guarducci, Marco Piccardi e Leonardo Rombai - ha prodotto il sito web www.toscanatirrenica.it e i due volumi sul litorale e sulle fortificazioni del 2012 e 2014, cui si fa qui ampio riferimento. 


\section{Gli studi}

Riguardo alla letteratura qui considerata, a partire da quella originale, è doveroso ricordare l'avvio delle ricerche sulle fortificazioni dei Presidios orbetellani e della costa maremmana concretizzatosi, fra gli anni ' 70 e ' 80 del XX secolo, ad opera di: Leonardo Rombai e Gabriele Ciampi (1979), Maurizio De Vita (1979 e 1980), Enrico Coppi (1982; Coppi e Rombai (1988), Danilo Barsanti, Dino Bravieri e Leonardo Rombai (a cura di, 1988). E ciò, dopo - o in contemporanea a - gli studi pionieristici di Giuseppe Caciagli sullo Stato dei Presidi (1971), di Paolo Cammarosano e Vincenzo Passeri sui castelli e sulle strutture fortificate dell'antico Stato Senese (1984) e di Giuseppe Guerrini e Massimiliana Catena per quelle della Provincia di Grosseto (1986).

La frammentazione politico-amministrativa che, dalla fine del XIII secolo in poi, interessò la Toscana costiera e insulare spiega: la dispersione delle fonti documentarie in centri di conservazione toscani (Massa e Carrara, Lucca, Firenze, Pisa, Piombino, Siena, Grosseto, Orbetello, ecc.), italiani (Genova, Modena, Roma e Napoli) e stranieri (della Spagna, della Francia e dell'Austria); il carattere locale o subregionale degli studi; e l'assenza quasi assoluta di lavori d'insieme sulle fortificazioni, con l'eccezione del non sempre affidabile volume di Baggiossi (1988) e degli scritti più documentati e innovativi - anche per l'impostazione catalografica moderna - di Principe (1988) riguardo al territorio del Granducato, di Manetti (1991) e di Taddei e Corazzi (2004) riguardo al litorale continentale. Apprezzabili per metodologie e risultati conoscitivi risultano i più recenti studi di Errico e Montanelli per il Livornese (2005), di Romanelli per il Livornese-Grossetano (2002), di Carrara per il Piombinese (2000) e di Della Monica, Roselli e Tosi per l'Argentario, l'Orbetellano e le isole di Giglio e Giannutri (1996).

Dagli anni '70 e '80 e fino all'inizio del nuovo millennio si sono dunque poste le basi per la trattazione del tema complesso e multidisciplinare dei centri fortificati e delle singole fortificazioni (fortezze, torri o altri fabbricati con funzioni militari) della costa toscana: tema sul quale negli ultimi decenni sono stati prodotti tanti studi per singoli centri o insediamenti, oppure per piccoli territori (le micro regioni storico-culturali) nei quali è possibile ripartire la Toscana tirrenica, in virtù delle diverse vicende politico-amministrative che li hanno storicamente interessati fino all'Unità d'Italia: almeno a decorrere dalla crisi della potenza politico-economica pisana (che a cavallo del Mille aveva unificato quasi tutto l'arco costiero) e dalla conseguente rottura dell'unità dello spazio tirrenico toscano.

I riferimenti bibliografici già fatti e la rassegna che segue mettono in luce aspetti importanti, a partire dalla multidisciplinarietà della produzione che appartiene a svariate declinazioni disciplinari: la storica, la geografica, l'urbanistica, l'architettonica (o storico-architettonica), l'archeologica. Dall'analisi delle opere emergono anche le diverse finalità delle ricerche: la ricerca pura o il recupero/ristrutturazione delle architetture in funzione della valorizzazione economica e/o della tutela, esercitabili per conto del pubblico o del privato.

Riguardo al ricco universo delle opere territorialistiche precedenti l'Atlante citato (Guarducci, Piccardi e Rombai, 2012), ai fini della presente ricerca risultano preziosi alcuni studi regionali che abbracciano l'intera Toscana tirrenica: le memorie di viaggio e di governo dei granduchi Pietro Leopoldo e Leopoldo II d'Asburgo Lorena (Pietro Leopoldo, 1969-74; e Pesendorfer, a cura di, 1987); il dizionario geografico primo-ottocentesco di Emanuele Repetti (1833-46); la descrizione delle stazioni balneari della fine del XIX secolo di Guido Carocci (1900), l'inquadramento geografico curato da Claudio Greppi nel 1993; e, relativamente alle principali città murate e agli insediamenti portuali, rispettivamente il classico studio di Edoardo Detti, Gian Franco Di Pietro e Giovanni Fanelli del 1968 e il repertorio di Adriano Betti Carboncini del 2001.

Per le diverse subregioni della Toscana costiera e per l'arcipelago ormai ragguardevoli sono i 
fondamenti bibliografici generali ${ }^{1}$; del resto come per i singoli centri urbani e minori ${ }^{2}$.

Ovviamente, le opere sopra ricordate su singoli insediamenti e subregioni contengono riferimenti - anche sostanziosi - alla genesi e alle trasformazioni delle architetture fortificate, per le quali si dispone anche di non pochi studi specifici ${ }^{3}$.

Altri lavori, con progetti e anche consuntivi di interventi di recupero riguardano - in progressione cronologica - una quindicine di strutture fortificate a partire dagli anni ' 80 del XX secolo ${ }^{4}$.

\section{Le fonti documentarie}

Il lavoro di approfondimento da svolgere sul terreno comporta obligatoriamente l'analisi diretta delle evidenze materiali, la restituzione fotografica (e ove possibile il rilevamento grafico), la raccolta delle testimonianze orali e anche di documenti scritti e iconografici conservati localmente. Se gli immobili sono raggiungibili, o almeno osservabili da vicino - e non sempre questo è possibile, a causa del loro inserimento in proprietà private con accesso impedito agli estranei mediante chiusure delle vie che conducono alle strutture o a punti di visuale sulle medesime -, la ricerca diretta sul campo serve specialmente ad evidenziare la conformazione e le condizioni strutturali, lo stato di conservazione, la funzione, la proprietà o la gestione degli immobili; e, in definitiva, consente di effettuare la comparazione della realtà effettuale con gli assetti del passato prossimo e/o remoto certificati dalla documentazione storica disponibile.

Il lavoro sul terreno vale anche a svolgere approfondimenti e integrazioni di ricerca presso i proprietari e gli abitanti locali e, soprattutto, presso gli uffici comunali competenti, a partire dallo strumento urbanistico e dalle concessioni rilasciate o richieste dalla proprietà nel recente passato; senza ovviamente trascurare la biblioteca comunale che può conservare materiali editi e inediti, anche non catalogati, e quindi non reperibili presso le grandi biblioteche cittadine.
L'indagine diretta deve poi allargarsi agli uffici delle competenti soprintendenze per i beni architettonici, paesaggistici, storici, artistici ed etnoantropologici riguardo all'esistenza - come di regola esiste - di vincoli di tutela, e quindi di pratiche specifiche con documenti riguardanti il bene (paesistico, architettonico o archeologico) considerato. Infine, sarà utile condurre ricerche anche presso i collezionisti privati e le raccolte pubbliche di fotografie e cartoline postali d'epoca, oggi assai frequenti in virtù della bellezza o del fascino delle vedute delle fortificazioni, che spesso sono inquadrate in paesaggi arricchiti da scorci di litorale e di mare di sicuro effetto estetico. Queste ultime categorie di fonti iconografiche, le fotografie e cartoline postali (e più eccezionalmente le vedute e altre rappresentazioni proprie della pittura d'arte), hanno grande importanza perché possono essere agevolmente confrontate con i documenti di età precedente e/o successiva, oltre che ovviamente - con l'attualità, con conseguente attribuzione di significati circa i contenuti edilizi e funzionali delle strutture fortificate oggetto della ricerca.

L'indagine sistematica negli istituti di conservazione deve riguardare in primo luogo gli Archivi di Stato (sui quali ritorneremo), ma anche - e preliminarmente $-\mathrm{i}$ due enti cartografici ufficiali dello Stato italiano: l'Istituto Geografico Militare di Firenze (con la cartoteca che conserva la Carta d'Italia topografica alle scale di 1:100000, 1:50000 e 1:25000, nelle sue versioni cronologiche a partire dalla prima del tardo Ottocento, la biblioteca "Attilio Mori" che possiede carte dei secoli XVI-XIX, e la fototeca che dispone di foto aeree zenitali, a partire dagli anni ' 30 del XX secolo, con speciale importanza del volo GAI del 1954, il primo a coprire tutto il territorio nazionale); e l'Istituto Idrografico della Marina di Genova (per la cartografia nautica prodotta in varie versioni dalla fine del XIX secolo ad oggi). Altra tappa necessaria dell'indagine riguarda l'Ufficio del Catasto oggi Ufficio del Territorio, che possiede le mappe catastali aggiornate e quelle - particolarmente preziose - dell'impianto degli anni ' 30 e ' 40 del XX secolo in scala 1:2000, con anche i quadri d'insieme comunali in scala 1:20000. 
Anche la Regione Toscana - che dagli anni '70 del XX secolo produce la carta tecnica alle scale 1:10000 e 1:5000 - conserva non pochi voli, tra l'altro con figure meglio rivelatrici dei contenuti territoriali puntiformi per la quota di rilevamento assai più bassa (rispetto ai voli IGM) e talora anche per avere queste la caratteristica di rappresentazioni oblique anziché zenitali, utilissime per la rivelazione delle conformazioni murarie.

Come già anticipato, i contenitori di fonti documentarie più importanti sono gli Archivi di Stato delle attuali dieci province toscane e di altre regioni, a partire dagli istituti di quelle città esterne alla Toscana per cui gli Stati di appartenenza dell'età pre-unitaria hanno avuto poteri politico-amministrativi su territori oggi toscani: è il caso di Genova e di Torino (che raccolgono le memorie degli antichi Stati Ligure e Sabaudo) per l'isola di Capraia tra tardo Medioevo ed anni '20 del XX secolo; di Modena per buona parte dell'attuale provincia di Massa Carrara (Lunigiana e area Apuana) dalla metà del XVIII secolo all'unità d'Italia; di Napoli per l'Orbetellano e l'Argentario ed il centro elbano di Longone oggi Porto Azzurro che, dal 1557 al 1801, appartennero prima alla Spagna (con dipendenza dal Vicereame di Napoli) e poi al Regno Borbonico napoletano, salvo la parentesi austriaca del primo Settecento; per non parlare degli archivi di Parigi (Archivio di Stato e archivi militari delle armate di terra e di mare nel castello di Vincennes: Service Historique de l'Armée de Terre e de la Marine), dato che la Toscana fu dominata, direttamente o indirettamente, dal 1800 al 1814, dalla Francia napoleonica; e per non parlare, infine, del grande archivio romano dell'Istituto di Storia e Cultura dell'Arma del Genio, il quale conserva molti fondi stornati subito dopo l'Unità dagli archivi italiani. Ma, più in generale, le complesse vicende politico-militari dei territori e centri della Toscana tirrenica spiegano il perché importanti documentazioni sono oggi conservate in archivi statali della Spagna (Simancas e Madrid), dell'Austria (Vienna) e della Repubblica Ceca (a Praga esiste il grande Archivio Asburgo Lorena di Toscana (in Archivio Nazionale), costituito dopo l'espulsione del granduca Leopoldo II da Firenze il 27 aprile 1859 con la documentazione consegnata gradualmente all'ex sovrano e ai suoi emissari dai conservatori fiorentini.

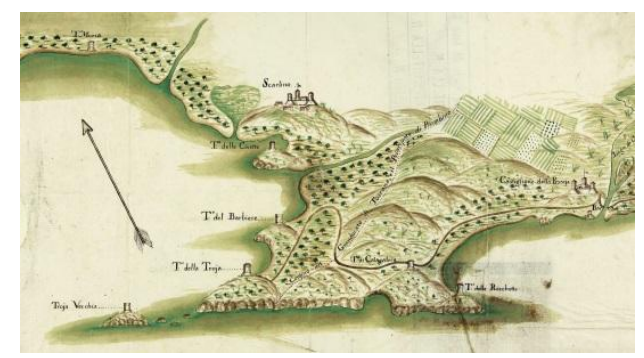

Fig. 1- Il promontorio di Punta Ala, 1780 ca. (Istituto Storico e di Cultura dell'Arma del Genio di Roma/ISCAG, 1230)

In Toscana e in Italia, ciascun archivio statale conserva - insieme a fondi di natura privata prodotti da famiglie, enti ed imprese del territorio di pertinenza - molteplici archivi che sono frutto dell'attività delle magistrature e degli uffici pubblici che si sono susseguiti, nel lunghissimo periodo compreso fra tempi medievali e contemporanei, nell'assetto amministrativo di uno o più Stati pre-unitari: di questi corpi di documenti è possibile avere un'idea preliminare attraverso gli specifici inventari a stampa o manoscritti, disponibili negli stessi istituti di conservazione e almeno in piccola parte evidenziati mediante i rispettivi siti internet, e attraverso l'elenco sintetico allegato allo scritto, che si allarga agli archivi comunali (v. cap. 4).

In ciascun istituto, di interesse primario risultano i materiali catastali storici sette-ottocenteschi e primo-novecenteschi - aggiornati fino al rilevamento del catasto vigente negli anni ' $30 \mathrm{e}$ '40 del XX secolo -, con le mappe in scala 1:2500 o 1:5000 (e con i centri abitati in scala $1: 1250$ ) e con i quadri d'unione comunali in scala variabile da 1:10000 a 1:60000. Le mappe sono accompagnate dai registri descrittivi (Tavole indicative e Campioni dei proprietari), indispensabili per una loro piena interpretazione contenutistica. Dagli Archivi di Stato, la ricerca deve allargarsi alle biblioteche delle grandi città e dei territori comunali considerati e ai tanti 
archivi comunali (oltre che ad altre conservatorie locali, pubbliche e private, ovviamente aperte alla consultazione) presenti nella Toscana tirrenica, i cui inventari, editi o inediti, sono depositati presso la Sovrintendenza Archivistica Regionale per la Toscana. A queste tipologie di conservatorie si è fatto ricorso nello studio delle torri e delle fortificazioni tirreniche: con utilizzazione di istituti come l'Archivio privato della famiglia Niccolini di Camugliano di Firenze e l'Archivio privato della famiglia Botti di Pietrasanta, l'Archivio Storico del Comune e la Biblioteca Civica Berio di Genova, la Biblioteca Giosuè Carducci di Pietrasanta, le Biblioteche Moreniana, Nazionale Centrale e Riccardiana di Firenze, la Bibliothéque Nationale de France di Parigi, il Gabinetto Disegni e Stampe della Galleria degli Uffizi di Firenze, la Österreichische Nationalbibliothek di Vienna, l'Archivio e la Biblioteca dell'Osservatorio Ximeniano di Firenze, il Laboratorio di Archeologia dei Paesaggi e Telerilevamento dell'Università degli Studi di Siena. Non è stata ignorata la ricerca in rete che (alle voci torri/fortezze/fortificazioni della costa toscana /livornese /del rossetano /dell'Argentario /dell'Elba, oltre che alle voci specifiche degli insediamenti) consente di ottenere dati e informazioni di ogni genere, ovviamente da vagliare criticamente con la massima cura.

Anche la bibliografia serve a chiarire - a grandi linee - le caratteristiche dei documenti considerati, scritti e grafici. Le rappresentazioni cartografiche disponibili per i secoli XV-XIX differenziate per linguaggi e qualità metriche, con prodotti più propriamente riferibili al vedutismo pittorico - costituiscono fonti assolutamente primarie. L'assunto è dimostrato, in primo luogo, da alcuni atlanti di cartografie sulle fortificazioni toscane, rilevate e ritratte dal genio militare lorenese (con il coordinamento del colonnello Odoardo Warren) nel 1749 e conservate nell'Archivio di stato di Firenze (Gurrieri, a cura di, 1979), e alle fortificazioni delle coste grossetane dell'architetto granducale Pietro Conti del 1793, atlante conservato nell'Osservatorio Ximeniano di Firenze
(Barsanti, Bravieri e Rombai, 1988); e dalle raccolte di disegni di coste e fortificazioni fatti dal mare, come le carte nautiche seisettecentesche della marina militare francese custodite negli archivi parigini (Guarducci, 2000, 2001 e 2010); il ben noto taccuino del cavaliere stefaniano Ignazio Fabroni, riempito tra 1664 e 1687 e conservato nella Biblioteca Nazionale Centrale di Firenze, utilizzato a più riprese per caratterizzare Livorno, Portoferraio e l'Elba e l'Argentario (Mazzanti, 1939 e 1940; Battaglini, 1981; Leoni Zanobini, 1987; Della Monica, Roselli e Tosi, 1994); e quello meno conosciuto su Portoferraio del governatore Mario Tornaquinci del 1688-1701, conservato nella Biblioteca Moreniana di Firenze (Lamberini e Manetti, 1981).

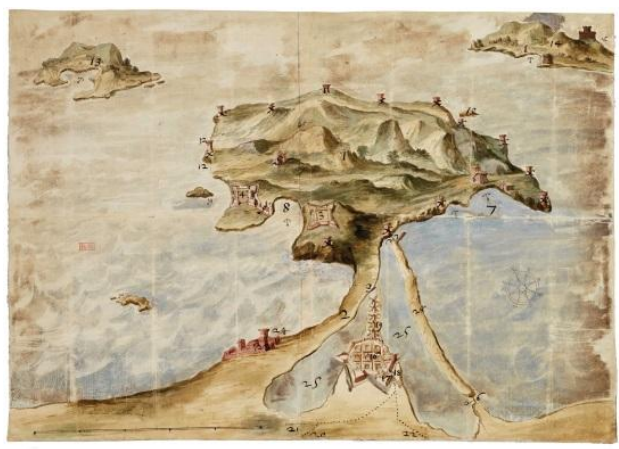

Fig. 2- Il Monte Argentario, Orbetello e le isole (Bibliothèque Nationale de France, Marine, 82bis, 17).

Riguardo alla cartografia, non mancano i repertori che riuniscono rappresentazioni di varia età sulla Toscana tirrenica e che sono conservati in un unico archivio: come quelli storici della Biblioteca "Attilio Mori" dell'Istituto Geografico Militare di Firenze (Catalogo ragionato, 1934) e il Portolano dell'Istituto Idrografico della Marina di Genova (Istituto Idrografico, 1947). Molte delle cartografie e delle foto aeree istituzionali più recenti sono state poi utilizzate e riprodotte nell'Atlante diacronico delle coste toscane: anni Cinquanta-anni Ottanta (Regione Toscana, 1996).

Altre opere, anche scritte, riguardano l'Istituto Storico e di Cultura dell'Arma del Genio di 
Roma (Fara, Conforti e Zangheri, 1978; Coppi e Rombai, 1988); l'Archivo General de Simancas (Principe e Romby, 1982; Tognarini, 1986; e Fanciulli, 1999); l'Archivio Asburgo Lorena di Toscana nell'Archivio Nazionale di Praga (Archivio di Stato di Firenze, 1991; Bonelli Conenna, a cura di, 1997); l'Archivio di Stato di Parigi e gli archivi militari di Vincennes della stessa capitale (Rombai, Quaini e Rossi, 1995; Rossi, 2000; Guarducci, 2000 e 2001); per il Pisano, i fondi Ufficio Fiumi e Fossi e Corpo degli Ingegneri di Acque e Strade del Compartimento Pisano, conservati nell'Archivio di Stato di Pisa (rispettivamente Barsanti, 1987; e Amico, 1995); per il Grossetano, il fondo Ufficio dei Fiumi e Fossi dell'Archivio di Stato di Grosseto (Bueti, 1980 e 1985); per l'intero litorale, la Miscellanea di Piante e, per l'isola d'Elba, lo Scrittoio delle Fortezze e Fabbriche, fondi conservati nell'Archivio di Stato di Firenze (rispettivamente Rombai, Toccafondi e Vivoli, a cura di, 1987; e Peria e Ferruzzi, a cura di, 2010); per l'isola di Capraia, il fondo Pretura di Capraia, conservato nell'Archivio di Stato di Genova (Brizi, 2012).

Non sono da dimenticare l'archivio della famiglia fiorentina Salviati, conservato nella Scuola Normale Superiore di Pisa (Karwacka Codini e Sbrilli, a cura di, 1987); la cartografia nautica tardo-medievale e moderna (Guarnieri, 1932) e il celebre atlante nautico Kitab-IBahariye del 1521-25 dell'ammiraglio turco Piri Reis (Lepore, Piccardi e Pranzini, 2011).

Studi degli ultimi decenni hanno fatto conoscere corpi di cartografie presenti in archivi $\mathrm{e}$ biblioteche soprattutto (ma non solo) toscani, con stretto riferimento a magistrature e uffici che li produssero (Guarducci, a cura di, 2006). Il comune denominatore di tali corpi miscellanei è il riferimento a questo o quel territorio: i repertori che li riguardano sono stati approntati per effettuare studi fini a se stessi, eventi espositivi o archivi digitali, come in generale ben dimostrano il database toscanatirrenica.it e l'Atlante della Toscana tirrenica (Guarducci, Piccardi e Rombai, 2012), insieme a numerosi cataloghi e volumi ${ }^{5}$.

\section{Appendice. Conservatorie toscane}

a) Archivi di Stato della Toscana

Catasti/decime/estimi descrittivi (secc. XIVXVIII); Catasti geometrici: lorenese, borbonico lucchese, estense massese; Archivi di Magistrature e Uffici preposti al governo/controllo del territorio in età preunitaria: in ciascun AS toscano (leggi e normative, memorie descrittive, piani e progetti, cartografia, personale addetto...). Soprattutto: a Firenze, Mediceo del Principato, Miscellanea medicea, Capitani di Parte Guelfa, Segreteria di Gabinetto, Segreteria di Gabinetto Appendice, Scrittoio delle Fortezze e Fabbriche (Medicee e Lorenesi), Segreteria di Finanze, Confini, Magona, Principato di Piombino, Acque e Strade; a Lucca, Capitoli, Riformagioni della Repubblica, Offizio sopra la Maona e Foce di Viareggio, Offizio sopra le differenze dei confini, Guardia di Palazzo, Beni e fabbriche pubbliche, Segreteria di Stato e Gabinetto, Acque e Strade; a Pisa, Ufficio Fiumi e Fossi, Acque e Strade, Prefettura; a Livorno, Dipartimento del Mediterraneo, Acque e Strade, Prefettura; a Siena, Ufficiali su Casseri e Fortezze, Kaleffo vecchio, Concistoro, Quattro Conservatori, Dipartimento dell'Ombrone; a Grosseto, Ufficio Fiumi e Fossi, Acque e Strade, Prefettura;

Cartografia, fondi specifici; Visite amministrative (a Stato Senese e Maremma, boschi, opifici della siderurgia, opifici delle saline, ecc.), inchieste e censimenti: in età preunitaria;

Archivi enti religiosi, ospedalieri, cavallereschi soppressi e/o espropriati; Uffici di gestione patrimoni edilizi-fondiari statali (fortificazioni e fabbricati civili, strutture di controllo doganale e sanitario del territorio, beni agricolo-forestali, opifici e miniere);

Notarili;

Diplomatici; Prefettura (dal 1861 all'ultimo dopoguerra); Archivi privati familiari e di enti ospedalieri, religiosi e cavallereschi: in ciascun AS toscano, documenti di famiglia e di gestione di imprese o di patrimoni edilizi-fondiari. 


\section{b) Archivi Comunali}

Statuti; Atti amministrativi e deliberativi (Consiglio, Giunta);

Atti fiscali (tassa prediale, tassa di famiglia, dazzaioli); Lavori pubblici: in età preunitaria, Provveditori di Strade, Acque e Fabbriche pubbliche, Ingegneri di Acque e Strade; in età unitaria, Ufficio Tecnico (strade comunali e vicinali, acque pubbliche, edilizia pubblica e privata con piazze e mercati);

Piani edilizi e urbanistici: in età unitaria;

Censimenti (età unitaria);

Inchieste economiche (età unitaria);

Anagrafe e stato civile (età unitaria.

\section{Note}

(1) Sono rappresentati da Mazzanti e Pasquinucci (1983) e da Azzari (1993) per la costa settentrionale compresa tra il fiume Magra e Livorno; dal ricchissimo lavoro di Giampaoli (1984) per l'area apuana; da Nepi e Mazzei, a cura di (2001), Nepi (2003), Bertuccelli Migliorini e Caccia, a cura di (2006), Azzari, Guarducci e Rombai (2006), Belli e Nepi, a cura di (2014) per la Versilia di Pietrasanta e/o di Viareggio; da Cervellati e Maffei Cardellini, a cura di (1988), Mazzanti, a cura di (1994), Severini (1999), Ciuti, a cura di (2005) e Micheletti (2011) per il litorale pisano a nord e a sud dell'Arno; da Mazzanti (1982) per il litorale livornese; da Bortolotti (1976) per la Maremma settentrionale; da Cappelletti (1897), Romero (1986), Azzari e Rombai (1986), Comune di Piombino, a cura di (1995), Carrara (1996) per il litorale piombinese; da Barsanti et alii (1982), Guarducci, Kukavicic, Piccardi e Rombai (2011) per il litorale grossetano e - per il parco della Maremma - da Guerrini (1981), Ciuffoletti e Guerrini, a cura di (1989) e Innocenti (1998); da Capezzuoli (1981 e 1982), Capezzuoli e Cavina (1981), Ferretti, a cura di (1982), Cavoli (1988), D'Anna (1989), Grasso (1994-95), Bufardeci (1995-96), Vichi (1996), Fanciulli (1999), Tognarini (2000), Guarducci, a cura di (2000 e 2003) per il litorale orbetellano e l'Argentario (Stato dei Presidi). Riguardo all'Arcipelago, fra le opere più utili si segnalano i lavori generali di Naldi e Pedicchio (1989) e di Muscari e Cunico (2012) e quello sulle colonie penali di Gambardella (2009). Le varie isole dispongono degli scritti di Errico e Montanelli (2000) per Gorgona; di Riparbelli (1973) e di Brizi (2005) e Moresco (2003, 2007 e 2008) per Capraia; di Ninci (1898), Lambardi (1981) e Canestrelli (1998) per l'Elba; di Foresi (1842/1999), Chierici (1875), Foresi e Piga (2001), Mastragostino (2001) e Mazzei, Braschi e Foresi (2004) per Pianosa; di Angelelli (1903), Michel (1925), Lopes Pegna (1953), Baccetti (1977) e Camici (2008 e 2010) per Montecristo; di Cavoli (1988), Roani Villani (1993) e Begnotti (1999, 2006, 2009 e 2010) per Giglio e Giannutri.

(2) Carrara e Marina di Carrara sono studiati da Bernieri, Mannoni e Mannoni (1983) e Giorgieri (1992); Massa e Marina di Massa da Bertozzi (1985); Pietrasanta da Buselli, 1970 e Maccari, 2003; Forte dei Marmi da Barberi (1950), Giannelli (1970), Il Forte dei Marmi (1997), Tenerini e Belli, a cura di (1997), Belli e Nepi (2005) e Buselli e Paolicchi (2009); Viareggio da Lenci (1941), Lopes Pegna (1958), Bergamini e Palmerini (1964), Bensio (1986), Fanfani (2005) e Cecchetti (2006); Marina di Pisa/Bocca d'Arno da Bertelli (1995); Livorno da Atti del convegno Livorno e il Mediterraneo (1978) e Matteoni (1985); San Vincenzo da Pazzagli (2011); Populonia da Fedeli (1983); Piombino da Ghelardoni (1977) e Tognarini e Bucci (1978); Follonica da Rombai e Tognarini (1986) e Saragosa (1995); Castiglione della Pescaia da Barsanti (1984); Marina di Grosseto da Innocenti (1999); Talamone da Cherubini (1973), Baglioni (1984), Scoppola (1983), Guidoni e Pieroni (1994), Sordini (2000) e Friedman (2010); Porto Santo Stefano da Fanciulli (1970); Orbetello da Fanciulli (1974), Broglia, a cura di (2001) e Federici (2009); Portoferraio (specificamente per la genesi di città fortificata cinquecentesca e sue trasformazioni) da Battaglini (1978 e 1980), Manetti (1979, 1995 e 1996), Lamberini e Manetti e altri (1981) e Fara (1997); Longone/Porto Azzurro (per la genesi di città fortificata primo secentesca e sue 
trasformazioni) da Vadi (1977) e - per Forte Focardo - Trotta (1987).

(3) Quali Buselli (1970) e Maccari (2003) per la terra murata di Pietrasanta con le sue rocche; Bini (1960) e Pelù (1974) per il forte di Motrone; Baroni e Gorreri (2005) per le trequattro strutture fortificate di Bocca di Serchio; Piancastelli Politti Nencini (1995) e Vaccari, a cura di (2002) per le due fortezze medicee di Livorno; Taddei et alii (1982) per il castello di Piombino; Vellati (1999) per il forte delle Marze; Vellati (1999) per la torre della Trappola; Bueti, Fommei e Vellati (1995), Fommei e Vellati (1996), Vellati (1999) per il forte di San Rocco; Maioli Urbini (1994) e Vellati (1999) per le torri dei Monti dell'Uccellina; Maioli (2000), Russo (2002) e Martinelli (2006) per le fortificazioni dei Presidios; Luzzetti (1974) per la rocca di Porto Ercole, con Coppi (1982) che allarga l'analisi anche ai vicini forti Filippo e Stella.

(4) E' il caso del fronte bastionato di Orbetello (Maioli Urbini, 1982 e 1984),deil forte delle Saline d'Albegna (Maioli Urbini, 1984), della rocca pisana del Giglio (Maioli Urbini, 1986), delle torri elbane di San Giovanni in Campo (Ricci, 1990), di Rio Marina (Gimma, 2009) e di Marciana Marina (con progetto redatto da Giuseppe A. Centauro dell'Università di Firenze dal 2012-13 in poi), della torre dell'Argentiera dell'Argentario (Della Monica, Roselli e Tosi, 1995), del Casale Spagnolo del tombolo della Giannella (Della Monica, Roselli e Tosi, 1997), della torre del Salto alla Cervia (Godino, Nicoliello e Torsello, 2001-2003), del forte Stella di Porto Ercole (Maioli, 2002), del forte del Pozzarello di Porto Santo Stefano (Della Monaca, Giordano e Metrano, 2002), della torre di Castel Marino (Maioli Urbini, 2009), della torre di San Vincenzo (Morandini, 2010) e del castello-forte di San Giorgio a Capraia (Brizi, 2012; Milanese, Febbraro e Meo, 2012).

(5) Per i Presidios di Orbetello (Rombai e Ciampi, 1979; Bueti, 1990; Rombai, Quaini e Rossi, 1995; Rossi, 2000; Guarducci, 2000 e 2001; Guarducci e Rombai, 2009); e altri per il litorale compreso fra il fiume Magra e Rosignano (Mazzanti, 1982; Mazzanti e Pasquinucci, 1983; Mazzanti e Pult Quaglia, 1986; Mazzanti e Sbrilli, 1991); per la Maremma grossetana (Barsanti, Bonelli Conenna e Rombai, 2001); per il Principato di Piombino (Comune di Piombino, a cura di, 1995); per la pianura di Pisa (la cartografia: Guarducci, Piccardi e Rombai, 2009; e le opere pittoriche otto-novecentesche: Scardino, a cura di, 1995); per l'isola di Capraia (Moresco, 2008); e per la città di Livorno (Frati, 2000).

\section{References}

Per il dettaglio dei testi utilizzati e citati si rimanda alla bibliografia pubblicata su www.toscanatirrenica.it.

Guarducci A., Piccardi M., Rombai L. (2012) Atlante della Toscana tirrenica. cartografia, storia, paesaggi, architetture. Livorno. Debatte Editore.

Guarducci A., Piccardi M., Rombai L. (2014) Torri e fortezze della Toscana Tirrenica. Storia e beni culturali. Livorno. Debatte Editore. 